\title{
Effect of Oscillator Phase Noise on Uplink Performance of Large MU-MIMO Systems
}

Antonios Pitarokoilis, Saif Khan Mohammed and Erik G. Larsson

\section{Linköping University Post Print}

N.B.: When citing this work, cite the original article.

(C2012 IEEE. Personal use of this material is permitted. However, permission to reprint/republish this material for advertising or promotional purposes or for creating new collective works for resale or redistribution to servers or lists, or to reuse any copyrighted component of this work in other works must be obtained from the IEEE.

Antonios Pitarokoilis, Saif Khan Mohammed and Erik G. Larsson, Effect of Oscillator Phase Noise on Uplink Performance of Large MU-MIMO Systems, 2012, Proceedings of the 50th Annual Allerton Conference on Communication, Control, and Computing.

Postprint available at: Linköping University Electronic Press http://urn.kb.se/resolve?urn=urn:nbn:se:liu:diva-80178 


\title{
Effect of Oscillator Phase Noise on Uplink Performance of Large MU-MIMO Systems
}

\author{
Antonios Pitarokoilis, Saif Khan Mohammed, Erik G. Larsson
}

\begin{abstract}
The effect of oscillator phase noise on the sum rate performance of a frequency selective multi-user multipleinput multiple-output (MU-MIMO) uplink channel is studied under imperfect channel state information. A maximum ratio combining detection strategy is employed by the base station (BS) (having a large antenna array of $M$ elements), and an analytical expression of a lower bound on the sum capacity of the system is derived. It is shown that an array power gain of $O(\sqrt{M})$ is achievable. It is also observed that phase noise effectively limits the fraction of the time used for information transmission and the number of users in the system. Finally it is concluded that, phase noise degrades the performance but does not eliminate the fundamental gains of a Large Scale Antenna System (LSAS), i.e., power efficiency and high sum rate performance with low complexity receiver processing.
\end{abstract}

\section{INTRODUCTION}

Multi-user multiple-input multiple-output (MU-MIMO) systems have been shown to provide an attractive solution to the ever increasing demand for high data rates in cellular wireless networks [1]. At the same time, it is necessary to increase energy efficiency in communication networks. Studies towards this direction have shown that the use of unlimited number of base station (BS) antenna elements and low complexity linear transceiver techniques can provide unprecedented multiplexing and array power gains [2]. In [3] it is proved that single-cell Large Scale Antenna Systems (LSAS) can provide $O(M)$ and $O(\sqrt{M})$ array power gains ${ }^{1}$ for the case of flat fading uplink with perfect and imperfect channel state information (CSI), respectively, where $M$ is the number of BS antennas. A similar array gain of $O(M)$ is shown to hold also for the frequency selective MU-MIMO downlink channel with perfect CSI [4].

At the transmitter chain, after the baseband processing the information signal is up-converted to passband by multiplication with the carrier generated by a local oscillator. The phase of this carrier signal varies randomly with time, thereby distorting the information signal. Similar distortion is present in the receiver chain during down-conversion from

The authors are with the Department of Electrical Engineering (ISY), Linköping University, 58183 Linköping, Sweden, \{antonispit, saif, erik.larsson\}aisy.liu.se

This work was supported by the Swedish Foundation for Strategic Research (SSF) and ELLIIT. E. G. Larsson is a Royal Swedish Academy of Sciences (KVA) Research Fellow supported by a grant from the Knut and Alice Wallenberg Foundation. The work of Saif K. Mohammed is supported in part by the Center for Industrial Information Technology (CENIIT), Linköping University, Sweden.

${ }^{1} \mathrm{An} O(\sqrt{M})$ array power gain implies that, for a fixed desired per user spectral efficiency, the per user transmit power can be reduced by $1.5 \mathrm{~dB}$ for every doubling in the number of BS antennas while maintaining a constant information rate to each user. passband to baseband. The phenomenon of phase noise is a non-trivial impairment in communication systems and it cannot be easily estimated and compensated for. Hence, significant research has been conducted for the assessment and mitigation of the effect of phase noise [5], [6], [7], [8]. However, the work presented in this paper is the first, to the authors' knowledge, to address the issue of phase noise in the uplink of frequency selective LSAS, where low complexity detection and obtaining reliable channel estimates is a challenge due to the large number of BS antennas.

The main contributions of this paper can be summarized as follows. 1) Firstly, we propose a low-complexity channel estimation and detection scheme for the uplink of a frequency selective multi-user LSAS in the presence of phase noise, 2) for the proposed schemes, a closed form expression for an achievable information sum-rate is derived. Analysis of the information rate expression reveals that, even with the proposed simple channel estimation and detection schemes, an $O(\sqrt{M})$ array gain is achievable in the presence of phase noise, 3) even though significant array gain can be achieved, the loss in information rate performance (when compared to a system with no phase noise) can be significant specially when the desired spectral efficiency is large. Our study however reveals that for low to moderate per-user spectral efficiency (around $1 \mathrm{bpcu}$ ) the loss in performance is small. 4) Another interesting aspect is as follows. Previous studies on the uplink information sum-rate for systems with no phase noise have revealed that the sum-rate increases with increasing number of users. However, interestingly, with phase noise and the proposed channel estimation/detection scheme, we observe that the information sum rate can decrease with increase in the number of users.

\section{System MOdEL}

We consider a frequency selective MU-MIMO uplink channel with $M$ BS antennas and $K$ single antenna users. The channel between the $k$-th user and the $m$-th BS antenna is modelled as a finite impulse response (FIR) filter with $L$ equally spaced channel taps. The $l$-th channel tap is given by $g_{m, k, l} \triangleq \sqrt{d_{k, l}} h_{m, k, l}$, where $h_{m, k, l}$ and $d_{k, l}$ model the fast and slow time varying components, respectively. In this paper we assume a block fading model where $h_{m, k, l}$ is fixed during the transmission of a block of $K L+N_{D}$ symbols and varies independently from one block to another. $N_{D}$ denotes the number of channel uses utilized for data transmission (see Fig. 1). $d_{k, l} \geq 0, l=0, \ldots, L-1$ models the power delay profile (PDP) of the frequency selective channel for the $k$-th 
user. Since $\left\{d_{k, l}\right\}$ vary slowly with time, we assume them to be fixed for the entire communication. We further assume $h_{m, k, l}$ to be i.i.d. $\mathcal{C N}(0,1)$ distributed. Further, the PDP for every user is normalized such that the average received power is same irrespective of the length of the channel impulse response. Therefore, it holds

$$
\sum_{l=0}^{L-1} \mathbb{E}\left[\left|\sqrt{d_{k, l}} h_{m, k, l}\right|^{2}\right]=\sum_{l=0}^{L-1} d_{k, l}=1,
$$

$1 \leq k \leq K$. Finally, we assume exact knowledge of the channel statistics at the BS, but not of the particular channel realizations.

\section{A. Phase Noise Model}

Phase noise is introduced at the transmitter during upconversion, when the baseband signal is multiplied with the carrier generated by the local oscillator. The phase of the generated carrier drifts randomly, resulting in phase distortion of the transmitted signal. A similar phenomenon also happens at the receiver side during down-conversion of the bandpass signal to baseband. In the following, $\theta_{k}, k=$ $1, \ldots, K$ denotes the phase noise process at the $k$-th user and $\phi$ denotes the phase noise process at the $M$ BS receivers. The latter implies identical phase noise processes at the BS antenna elements, i.e. we assume full coherency between the BS receivers. This models the practical scenario of a centralized BS with a single oscillator output feeding the down-conversion module in each receiver. We further assume that the phase noise processes $\phi, \theta_{k}, k=1, \ldots, K$ are mutually independent. In this study every phase noise process is modelled as an independent Wiener process, which is a well-established model [9], [10]. Therefore, the discrete time phase noise process at the $\mathrm{BS}$ antennas at time $n$ is given by $^{2}$

$$
\phi[n]=\phi[n-1]+w[n],
$$

where $w[n] \sim \mathcal{N}\left(0,4 \pi^{2} f_{c}^{2} c T_{s}\right)$ are independent identically distributed zero-mean Gaussian increments. $f_{c}$ is the carrier frequency, $T_{s}$ is the symbol interval and $c$ is a constant that depends on the oscillator. Similarly, we can define the phase noise processes at the $K$ users.

\section{B. Received Signal}

Let $x_{k}[i]$ be the symbol transmitted from the $k$-th user at time $i$. The received signal at $m$-th BS antenna element at time $i$ is then given by

$$
y_{m}[i]=\sqrt{P} \sum_{k=1}^{K} \sum_{l=0}^{L-1} e^{-j \phi[i]} g_{m, k, l} e^{j \theta_{k}[i-l]} x_{k}[i-l]+n_{m}[i],
$$

where $n_{m}[i] \sim \mathcal{C N}\left(0, \sigma^{2}\right)$ is additive white Gaussian noise (AWGN). Each user transmits a stream of i.i.d. $\mathcal{C N}(0,1)$

\footnotetext{
${ }^{2}$ The discrete-time phase noise model is used since we are interested in the discrete-time complex baseband representation of the transmit and receive signals.
}

1 Transmission Block

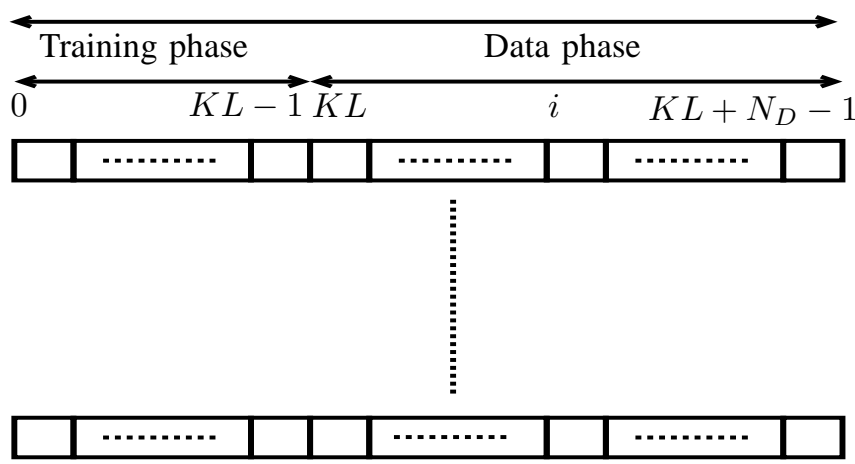

Fig. 1. Transmission schedule: The channel is assumed to be static during one transmission block. In each block, the first $K L$ channel uses are utilized for channel estimation (via uplink pilots) and the remaining $N_{D}$ channel uses are utilized for data transmission.

information symbols (i.e. $x_{k}[i] \sim \mathcal{C N}(0,1)$ ), that are independent of the information symbols of the other users. $P$ denotes the average uplink transmitted power from each user.

\section{TRAnSmission Schedule AND RECEIVE PROCESSING}

Motivated by the need for low-complexity channel estimation and detection algorithms, we propose the following block based uplink transmission scheme. In the proposed scheme, a transmission block of $K L+N_{D}$ channel uses consists of $K L$ channel uses (for uplink channel estimation) followed by the data phase (for data transmission) of duration $N_{D}$ channel uses.

\section{A. Channel Estimation}

For coherent demodulation, the BS needs to estimate the uplink channel. This is facilitated through the transmission of uplink pilot symbols during the training phase of each transmission block. The users transmit uplink training signals sequentially in time, i.e. at any given time only one user is transmitting uplink training signals and all other users are idle. To be precise, the $k$-th user sends an impulse signal of amplitude $\sqrt{P_{p} K L}$ at the $(k-1) L$-th channel use and is idle for the remaining portion of the training phase. Here, $P_{p}$ is the average transmit power by a user during the training phase. Therefore, using (3) the signal received at the $m$-th BS receiver at time $i=(k-1) L+l, l=0, \ldots, L-1, k=$ $1, \ldots, K$ is given by

$$
\begin{aligned}
y_{m}[i] & =y_{m}[(k-1) L+l] \\
& =\sqrt{P_{p} K L} g_{m, k, l} e^{-j \phi[(k-1) L+l]} e^{j \theta_{k}[(k-1) L]} \\
& +n_{m}[(k-1) L+l] .
\end{aligned}
$$

The proposed channel estimates are then given by

$$
\begin{aligned}
\hat{g}_{m, k, l} & =\frac{1}{\sqrt{P_{p} K L}} y_{m}[(k-1) L+l] \\
& =g_{m, k, l} e^{-j \phi[(k-1) L+l]} e^{j \theta_{k}[(k-1) L]} \\
& +\frac{1}{\sqrt{P_{p} K L}} n_{m}[(k-1) L+l] .
\end{aligned}
$$


We choose the proposed training sequence since it allows for a very simplistic channel estimation scheme at the BS. As expected, the channel estimate is distorted by the AWGN and by the phase noise at the transmitter and at the BS.

\section{B. Maximum Ratio Combining}

Using (3), the received signal during the data phase is given by

$y_{m}[i]=\sqrt{P_{D}} \sum_{k=1}^{K} \sum_{l=0}^{L-1} e^{-j \phi[i]} g_{m, k, l} e^{j \theta_{k}[i-l]} x_{k}[i-l]+n_{m}[i]$,

where $i=K L, \ldots, N_{D}+K L-1$ and $P_{D}$ is the per user average transmit power constraint during the data phase. Motivated by the need for low-complexity detection, we propose a maximum ratio combining (MRC) receiver. The MRC receiver reverses the received symbols, $y_{m}[i]$, in the time domain and convolves them with the complex conjugate of the estimated channel impulse response. Therefore, the detected symbol, $\hat{x}_{k}[i]$, is given by

$$
\hat{x}_{k}[i]=\sum_{l=0}^{L-1} \sum_{m=1}^{M} \hat{g}_{m, k, l}^{*} y_{m}[i+l] .
$$

\section{Achievable Sum Rate}

In this paper, we consider the information sum-rate as the relevant performance metric for quantifying the effects of phase noise. To this end, using (5) and (6), (7) can be further expressed as

$$
\hat{x}_{k}[i]=A_{k}[i] x_{k}[i]+\operatorname{ISI}_{k}[i]+\operatorname{MUI}_{k}[i]+\operatorname{AN}_{k}[i],
$$

where

$$
\begin{aligned}
A_{k}[i] & =\sqrt{P_{D}} \sum_{m=1}^{M} \sum_{l=0}^{L-1}\left|g_{m, k, l}\right|^{2} e^{-j(\phi[i+l]-\phi[(k-1) L+l])} \\
& \cdot e^{-j\left(\theta_{k}[(k-1) L]-\theta_{k}[i]\right)} \\
\operatorname{ISI}_{k}[i] & =\sqrt{P_{D}} \sum_{m=1}^{M} \sum_{l=0}^{L-1} \sum_{\substack{q=0 \\
q \neq l}}^{L-1} g_{m, k, l}^{*} g_{m, k, q} e^{j \phi[(k-1) L+l]} \\
& \cdot e^{-j \phi[i+l]} e^{-j\left(\theta_{k}[(k-1) L]-\theta_{k}[i+l-q]\right)} x_{k}[i+l-q] \\
\operatorname{MUI}_{k}[i] & =\sqrt{P_{D}} \sum_{m=1}^{M} \sum_{\substack{p=1 \\
p \neq k}}^{K-1} \sum_{l=0}^{L-1} \sum_{q=0}^{L} g_{m, k, l}^{*} g_{m, p, q} \\
& \cdot e^{-j(\phi[i+l]-\phi[(k-1) L+l])} e^{-j\left(\theta_{k}[(k-1) L]-\theta_{p}[i+l-q]\right)} \\
& \cdot x_{p}[i+l-q] \\
\mathrm{AN}_{k}[i] & =\sqrt{\frac{P_{D}}{P_{p} K L}} \sum_{m=1}^{M} \sum_{p=1}^{K} \sum_{l=0}^{L-1} \sum_{q=0}^{L-1} g_{m, p, q} e^{-j \phi[i+l]} \\
& \cdot e^{j \theta_{p}[i+l-q]} n_{m}[(k-1) L+l] x_{p}[i+l-q] \\
& +\sum_{l=0}^{L-1} \sum_{m=1}^{M} \hat{g}_{m, k, l}^{*} n_{m}[i+l] .
\end{aligned}
$$

$A_{k}[i] x_{k}[i]$ is the desired signal term for the $k$-th user, $\operatorname{ISI}_{k}[i]$ stands for the intersymbol interference for user $k$ at time $i$, caused due to the information symbols of the $k$-th user transmitted at the previous $(L-1)$ channel uses, $\operatorname{MUI}_{k}[i]$ denotes the multi-user interference due to the other users and finally $\mathrm{AN}_{k}[i]$ is an aggregate noise term that incorporates the effect of the imperfect channel estimation and the receiver AWGN noise, $n_{m}[i]$. In the following, we describe a method to derive an achievable information rate for the $k$-th user. Similar techniques have been used earlier in [11], [12]. In (8), we add and subtract the term $\mathbb{E}\left[A_{k}[i]\right]$, where the expectation is taken over the channel gains, $g_{m, k, l}$, and the phase noise processes, $\theta_{k}, \phi$. This results in the following equivalent representation

$$
\hat{x}_{k}[i]=\mathbb{E}\left[A_{k}[i]\right] x_{k}[i]+\mathrm{EN}_{k}[i],
$$

where $\mathrm{EN}_{k}[i] \triangleq\left(A_{k}[i]-\mathbb{E}\left[A_{k}[i]\right]\right) x_{k}[i]+\operatorname{ISI}_{k}[i]+\operatorname{MUI}_{k}[i]+$ $\mathrm{AN}_{k}[i]$, is the effective noise term. In (9) the detected symbol, $\hat{x}_{k}[i]$, is a sum of two uncorrelated terms (i.e. $\left.\mathbb{E}\left\{\left(\mathbb{E}\left\{A_{k}[i]\right\} x_{k}[i]\right)\left(\mathrm{EN}_{k}[i]\right)^{*}\right\}=0\right)$. The first term is the desired symbol multiplied by a constant. This constant is known at the BS since the BS has knowledge of the channel statistics. The importance of the equivalent representation in (9) is that the scaling factor of the desired information symbol is a known constant. The exact probability distribution of $\mathrm{EN}_{k}[i]$ is difficult to compute. However, its variance can be easily calculated given that the channel statistics is known at the BS. Therefore, (9) describes an effective single user single-input single-output (SISO) additive noise channel, where the noise is zero mean, has known variance and is uncorrelated to the desired signal term. From the expressions for $A_{k}[i]$ and $\mathrm{EN}_{k}[i]$ in (8) and (9) the mean value of $A_{k}[i]$ and the variance $\mathrm{EN}_{k}[i]$ is given by the following theorem.

Theorem 1: The mean value of $A_{k}[i]$ and the variance $\operatorname{Var}\left(\mathrm{EN}_{k}[i]\right) \triangleq \mathbb{E}\left[\left|\mathrm{EN}_{k}[i]-\mathbb{E}\left[\mathrm{EN}_{k}[i]\right]\right|^{2}\right]$ are given by

$$
\begin{aligned}
\mathbb{E}\left[A_{k}[i]\right]= & \sqrt{P_{D}} M e^{-4 \pi^{2} f_{c}^{2} c T_{s}(i-(k-1) L)}, \\
\operatorname{Var}\left(\mathrm{EN}_{k}[i]\right) & =P_{D} M^{2} P_{\mathrm{pn}}+P_{D} M K \\
& +\sigma^{2} M\left(1+\frac{P_{D}}{P_{p}}+\frac{\sigma^{2}}{K P_{p}}\right),
\end{aligned}
$$

where $P_{\mathrm{pn}} \triangleq \sum_{l=0}^{L-1} \sum_{l^{\prime}=0}^{L-1} d_{k, l} d_{k, l^{\prime}} e^{-4 \pi^{2} f_{c}^{2} c T_{s}\left|l-l^{\prime}\right|}-$ $e^{-8 \pi^{2} f_{c}^{2} c T_{s}(i-(k-1) L)}$.

Proof: See Appendix .

From the expressions above, it follows that $\mathbb{E}\left[A_{k}[i]\right]$ and $\operatorname{Var}\left(\mathrm{EN}_{k}[i]\right)$ depend on $i$ and are different for different $i=K L, \ldots, K L+N_{D}-1$. Subsequently we shall refer to the effective SISO channel in (9) as the $i$-th SISO channel. Hence, for a given $i \in\left\{K L, \ldots, K L+N_{D}-1\right\}$ the statistics of the $i$-th effective SISO channel is the same across different transmission blocks (i.e., for a given $i, \mathbb{E}\left[A_{k}[i]\right]$ and $\operatorname{Var}\left(\mathrm{EN}_{k}[i]\right)$ is the same for all transmission blocks). Also, for a given $i$ the effective noise term $\operatorname{EN}_{k}[i]$ is i.i.d. from one transmission block to another. This motivates us to consider $N_{D}$ channel codes for each user, one for each $i=K L, \ldots, K L+N_{D}-1$. At the $k$-th transmitter (user), the 
symbols of the $i$-th channel code $\left(x_{k}[i]\right)$ are transmitted only during the $i$-th channel use of each transmission block. Similarly, at the BS, for a given user, for each $i$ the received and processed symbols (i.e. $\hat{x}_{k}[i]$ ) across different transmission blocks are jointly decoded. Essentially, this implies that, at the BS we have $N_{D}$ parallel channel decoders for each user. We propose the above scheme of $N_{D}$ parallel channel codes for each user only to derive a lower bound on the achievable information rate. In practice, due to reasons of complexity, channel coding/decoding would not only be performed across different transmission blocks, but also across consecutive channel uses within each transmission block. ${ }^{3}$

We are now interested in computing a lower bound on the reliable rate of communication for each of the $N_{D}$ channel codes. For each $i=K L, \ldots, K L+N_{D}-1$ a lower bound on the information rate for the effective channel in (9) can be computed by letting $x_{k}[i]$ to be Gaussian distributed. With Gaussian distributed information symbols, it is known that the worst case uncorrelated noise (i.e. resulting in minimum information rate) is Gaussian distributed with the same variance as that of $\mathrm{EN}_{k}[i]$. Consequently, a lower bound on $I\left(\hat{x}_{k}[i] ; x_{k}[i]\right)$ (i.e. the mutual information rate for the $i$-th channel code for user $k$ ) is given by

$$
R_{k}[i]=\log _{2}\left(1+\frac{P_{D} M e^{-8 \pi^{2} f_{c}^{2} c T_{s}(i-(k-1) L)}}{P_{D} M P_{\mathrm{pn}}+P_{D} K+\sigma^{2}\left(1+\frac{P_{D}}{P_{p}}+\frac{\sigma^{2}}{K P_{p}}\right)}\right)
$$

Since no data transmission happens during the training phase, the overall effective information rate achievable by the $k$-th user is given by

$$
R_{k} \triangleq \frac{1}{K L+N_{D}} \sum_{i=K L}^{K L+N_{D}-1} R_{k}[i]
$$

The achievable sum rate is therefore given by

$$
R=\sum_{k=1}^{K} R_{k}=\frac{1}{K L+N_{D}} \sum_{k=1}^{K} \sum_{i=K L}^{K L+N_{D}-1} R_{k}[i] .
$$

In the following $\beta \triangleq \frac{P_{p}}{P_{D}}>0$ denotes the ratio between the per-user average transmit power during the training phase and that during transmission phase.

\section{RESUlts AND Discussion}

Throughout this section, the plots used to illustrate the main results assume that $T_{s}=0.1 \mu s, f_{c}=2 G H z, c=$ $4.7 \times 10^{-18}(\mathrm{rad} \cdot \mathrm{Hz})^{-1}$. The selected parameters correspond to typical values of a wideband wireless communication system, such as a WLAN IEEE 802.11 [13]. Further, the users have a common exponential power delay profile that is fixed throughout the entire communication and is given by $d_{k, l}=e^{-l} / \sum_{i=0}^{L-1} e^{-i}, 1 \leq k \leq K$. The length of the channel echo is also fixed at $L=20$. Finally, the constant of proportionality between $P_{D}$ and $P_{p}$ is fixed to $\beta=1$,

\footnotetext{
${ }^{3}$ This is because in practice the channel statistics of the effective channel
} in (9) does not change appreciably across a few consecutive channel uses. hence $P_{p}=P_{D}$. We note that the plots are generated by evaluating the expressions in Theorem 1, (12) and (14). These expressions hold for every choice of $\beta>0$ and PDP that satisfies (1). We start by stating two Propositions on the performance of the system in the low and the high SNR regime, respectively.

Proposition 1: In the low SNR regime, the performance loss due to phase noise is not significant for sufficiently small data phase block size $N_{D}$.

Proof: The sum rate of the system when phase noise is present is given by (14), where

$$
R_{k}[i]=\log _{2}\left(1+\frac{\frac{P_{D}}{\sigma^{2}} M e^{-8 \pi^{2} f_{c}^{2} c T_{s}(i-(k-1) L)}}{\frac{P_{D}}{\sigma^{2}} M P_{\mathrm{pn}}+\frac{P_{D}}{\sigma^{2}} K+\left(\frac{1+\beta}{\beta}+\frac{\sigma^{2}}{K \beta P_{D}}\right)}\right) .
$$

On the other hand, the sum rate for the no-phase-noise case can be derived from (12), (13) and (14) by considering the oscillator to be perfect (i.e., the oscillator constant $c=0$ ),

$$
\mathcal{R}=\frac{K N_{D}}{N_{D}+K L} \log _{2}\left(1+\frac{\frac{P_{D}}{\sigma^{2}} M}{\frac{P_{D}}{\sigma^{2}} K+\left(\frac{1+\beta}{\beta}+\frac{\sigma^{2}}{K \beta P_{D}}\right)}\right) .
$$

It is clear that in the low SNR regime, i.e. $P_{D} / \sigma^{2} \ll$ 1 , the dominating factor in the denominator in the argument of the $\log _{2}$ function is, in both cases, the term $\left(\frac{1+\beta}{\beta}+\frac{\sigma^{2}}{K \beta P_{D}}\right)$. Therefore for scenarios where $N_{D}$ is not very large, $e^{-8 \pi^{2} f_{c}^{2} c T_{s}(i-(k-1) L)} \approx 1$ and the performance loss compared to the no-phase-noise scenario is small.

In Fig. 2 the sum rate performance of the system, as given by (14), is plotted as a function of SNR $\triangleq \frac{P_{D}}{\sigma^{2}}$ for $N_{D}=$ [100 100010000$]$ with $M=100, K=10$. The sum rate achieved without phase-noise is also plotted for the sake of comparison. We observe that at low SNR, the loss in sum rate performance is insignificant for small $N_{D}=$ [100 1000], whereas the loss is significant for large $N_{D}=10000$. This observation supports the result in Proposition 1.

Proposition 2: Saturation in the High-SNR regime. In the presence of phase noise the effective information rate of the $k$-th user saturates to the value

$$
\begin{aligned}
R_{k}^{\infty} & =\lim _{\frac{P_{D}}{\sigma^{2}} \rightarrow \infty} R_{k} \\
& =\frac{1}{N_{D}+K L} \sum_{i=K L}^{N_{D}+K L-1} \log _{2}\left(1+\frac{M e^{-8 \pi^{2} f_{c}^{2} c T_{s}(i-(k-1) L)}}{M P_{\mathrm{pn}}+K}\right) .
\end{aligned}
$$

Proof: The result follows immediately from (12) and the definition of $R_{k}$ in (13).

The saturation of the achievable sum rate at high SNR (reported in Proposition 2) is also clear from Fig. 2. Note that the saturation in general is the effect of the specific MRC based detection scheme proposed earlier. For both the phase noise and the no-phase-noise scenarios, an increase in the transmit power leads to an increase of both the desired signal power and the MUI power. As a result, the sum rate performance saturates. Compared to the nophase-noise case, for the phase noise scenario an additional sum rate performance penalty is caused due to the factors 


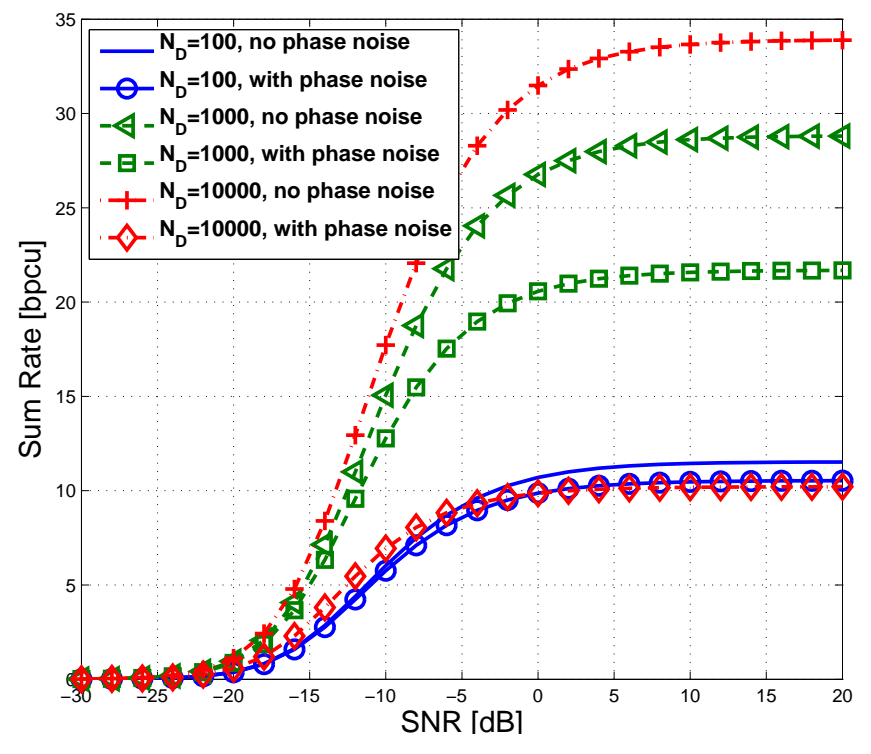

Fig. 2. Sum rate v.s. SNR for various values of $N_{D} . M=100, K=10$.

$e^{-8 \pi^{2} f_{c}^{2} c T_{s}(i-(k-1) L)}$ and $M P_{\mathrm{pn}}$ in (16).

Proposition 3: An $O(\sqrt{M})$ array gain is achievable for the frequency selective MU-MIMO uplink in the presence of phase noise and imperfect channel estimation, i.e. for a fixed number of users $K$, with a sufficiently large antenna array at the BS, the average transmitted power $P_{D}$ can be reduced by roughly $1.5 \mathrm{~dB}$ for every doubling in the number of $\mathrm{BS}$ antennas while maintaining a constant information rate for each user.

Proof: Set $P_{D}=E_{u} / \sqrt{M}$, where $E_{u}$ is fixed. By substitution in (13) we get

$$
\begin{aligned}
R_{k} & =\sum_{i=K L}^{N_{D}+K L-1} \frac{\log _{2}\left(1+\frac{\frac{E_{u}}{\sigma^{2} \sqrt{M}} M e^{-8 \pi^{2} f_{c}^{2} c T_{s}(i-(k-1) L)}}{\left(\frac{E_{u}}{\sigma^{2}} P_{\mathrm{pn}}+\frac{\sigma^{2}}{K E_{u} \beta}\right) M+T(M)}\right)}{N_{D}+K L} \\
& \stackrel{M \rightarrow \infty}{\longrightarrow} \sum_{i=K L}^{N_{D}+K L-1} \frac{\log _{2}\left(1+\frac{\frac{E_{u}}{\sigma^{2}} e^{-8 \pi^{2} f_{c}^{2} c T_{s}(i-(k-1) L)}}{\frac{E_{u}}{\sigma^{2}} P_{\mathrm{pn}}+\sigma^{2} /\left(K E_{u} \beta\right)}\right)}{N_{D}+K L},
\end{aligned}
$$

where $T(M) \triangleq \frac{1+\beta}{\beta} \sqrt{M}+\frac{E_{u}}{\sigma^{2}} K$. The fact that the limiting value of the rate is positive implies the $O(\sqrt{M})$ array power gain.

A significant property of large MIMO systems, is the array power gain that they offer, facilitating the design of highly power-efficient communication systems [2], [14], [4]. Proposition 3 extends this result to the case of phase-noiseimpaired large MU-MIMO systems. In Fig. 3 the sum rate performance is plotted over the number of BS antennas, $M$, for $K=10$ and $N_{D}=\left[\begin{array}{llll}100 & 500 & 10002000\end{array}\right]$, while the per user power is scaled as $P_{D}=E_{u} / \sqrt{M}$, where $E_{u}=1$ is fixed. The curves of the exact sum rate performance are compared with the $80 \%$ of their corresponding asymptotic values (computed by (17)). It is observed that the curves approach their asymptotic values at a slow rate, which can

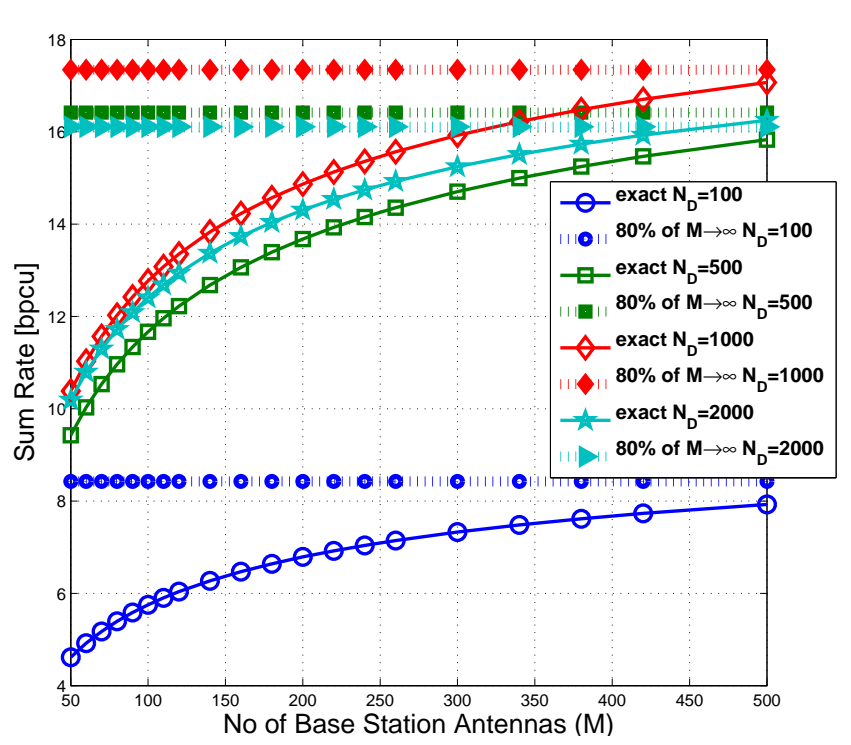

Fig. 3. Sum rate v.s. $M$ for various values of $N_{D}$ (fixed $K$ ). Per user transmit power $P_{D}=E_{u} / \sqrt{M}\left(E_{u}=1\right.$ is fixed).

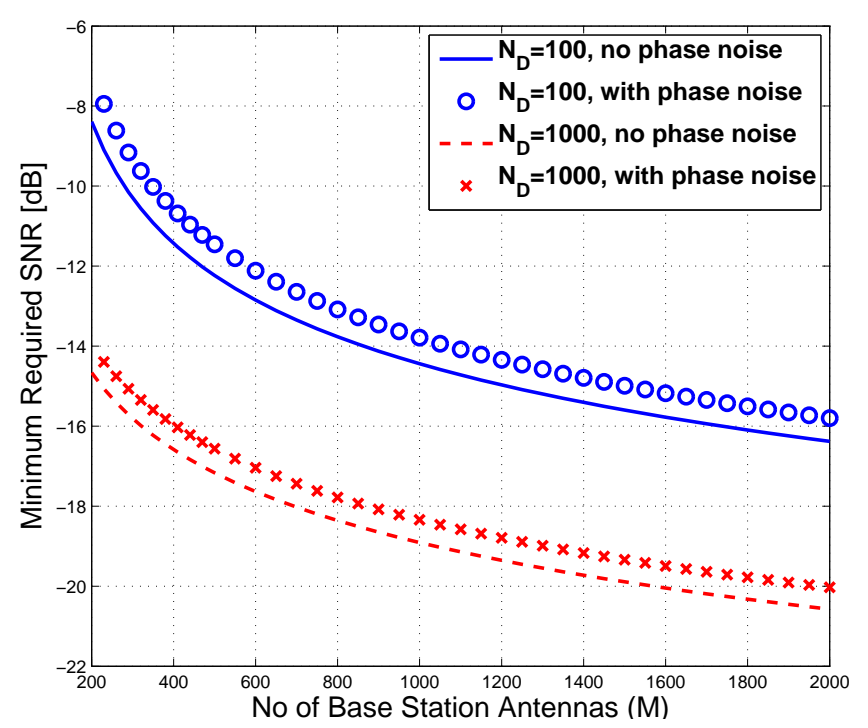

Fig. 4. Minimum required SNR for a fixed per user spectral efficiency of $r=1$ bpcu as a function of increasing $M$ (for various values of $N_{D}$ ). $K=10$ users.

\begin{tabular}{lcc}
\hline & \multicolumn{2}{c}{ Minimum Required SNR [dB] $N_{D}=1000$} \\
\cline { 2 - 3 } No of BS & No Phase Noise & Phase Noise \\
Antennas & & \\
\hline 400 & -16.58 & -15.96 \\
800 & -18.51 & -17.93 \\
1000 & -19.03 & -18.46 \\
1600 & -20.12 & -19.57 \\
2000 & -20.58 & -20.03 \\
\hline
\end{tabular}

TABLE I

Minimum Required SNR [DB] VS THE NUMber of BS ANTENNAS FOR $N_{D}=1000$. 
be explained by the fact that the dominating term of the denominator of the fraction inside the $\log _{2}(\cdot)$ expression in (17) is $O(M)$ whereas the remaining terms (see expression $T(M)$ in (17)) are $O(\sqrt{M})$. In the region where the curves approach their asymptotic value, it is true to say that one can scale down the per user transmit power by $\sqrt{2}$ (or $1.5 \mathrm{~dB})$ and at the same time double the number of BS antennas $M$ without compromising the spectral efficiency of the users. This justifies the term array power gain. The above observation is further supported through Fig. 4, where the minimum SNR (in $\mathrm{dB}$ ) required to achieve a fixed per user information rate of $r=1 \mathrm{bpcu}$ is plotted as a function of the number of BS antennas for $N_{D}=[1001000]$ and $K=10$. The plots for the phase-noise-free case are also given for the sake of comparison. In order to be more precise, we also tabulate in Table I representative values from Fig. 4 for $N_{D}=1000$. So, for example, when $N_{D}=1000$ an increase from $1000 \mathrm{BS}$ antennas to 2000 for the phase-noise-impaired systems yields a power gain of $(-18.46-(-20.03))=1.57 \mathrm{~dB}$. This number will asymptotically (as $M \rightarrow \infty$ ) approach the value $1.5 \mathrm{~dB}$.

Based on the previous results, illustrated in Figs. 2, 3 and 4 , it becomes clear that for fixed $M, K, L$ there is a fundamental trade-off between the length of the data interval, $N_{D}$, and the achievable sum rate performance. Since a fixed time interval of $K L$ channel uses is required for the channel estimation, a small data interval, $N_{D}$, leads to underutilization of the available resources, yielding a low sum rate performance. As $N_{D}$ increases, more resources are utilized for the data transmission increasing the sum rate performance. However, as it can be seen by (12), $R_{k}[i]<$ $R_{k}[i-1]$, which implies that the gain of increasing the data interval diminishes with increasing $N_{D}$. In fact, the individual rates $R_{k}[i]$ approach 0 as $i \rightarrow \infty$. Therefore, it is expected that beyond some critical value the rate that can be supported in the last channel uses of the transmission block will be insignificant. This phenomenon is caused due to the fact that with large $N_{D}$, the phase noise drift in the oscillators is so large such that there is a total loss of coherency between the received symbols during data phase and the estimated channel at the beginning of the transmission block.

In Fig. 5 the dependence of the sum rate performance on the length of the data interval, $N_{D}$, is plotted for $M=$ [50 1002505001000 ], SNR $=0 \mathrm{~dB}$ and $K=10$. It can be seen that, as expected, the sum rate initially increases with increasing $N_{D}$ up to a certain critical value of $N_{D}$, after which the sum rate decreases. Further, we also observe that this critical value of $N_{D}$ seems to be independent of the number of BS antennas. Therefore, we have the following remark.

Remark 1: Phase noise effectively limits the length of the data interval, $N_{D}$.

In another paper [4], for the downlink channel of a MUMIMO LSAS, we had observed that in the absence of phase noise, with maximum ratio transmission and perfect channel estimates at the BS, for a fixed $\mathrm{M}$ the sum-rate performance increases with increasing number of users $K$. In the uplink

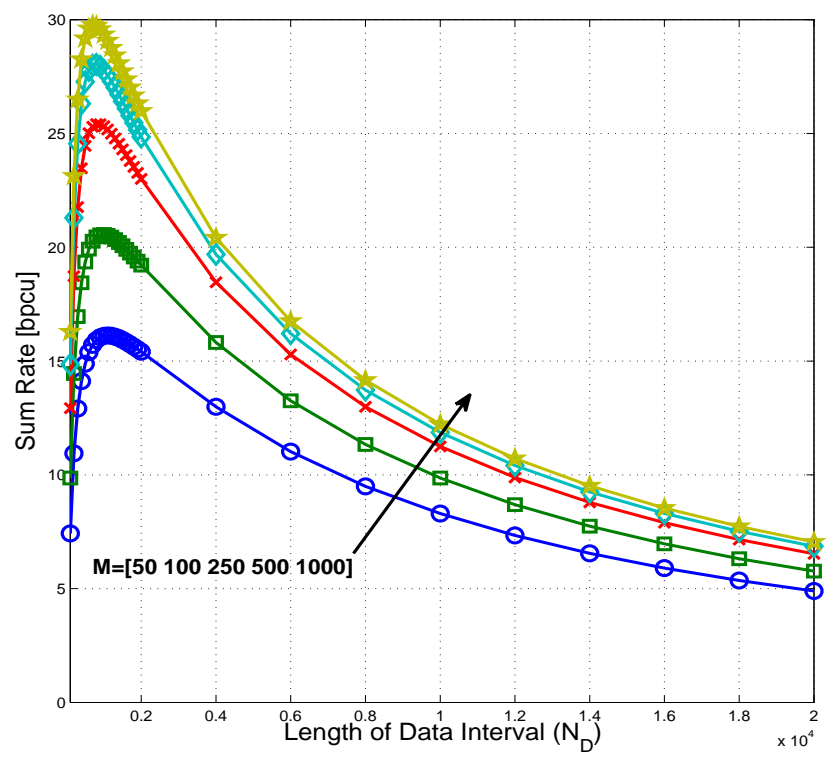

Fig. 5. Sum rate performance for increasing $N_{D}$, with fixed $M, K=10$, and $L=20$.

also, with no-phase-noise a similar behaviour is observed when MRC is performed with imperfect channel estimates. This can be observed in Fig. 6, where we fix the number of BS antennas to $M=100, \mathrm{SNR}=0 \mathrm{~dB}$ and plot the maximum achievable sum rate as a function of the number of the users $K$. For each value of $K$, we find the maximum achievable sum rate by numerically computing the optimal (critical) value of $N_{D}$, as shown in Fig. 7.

From the no phase noise curve in Fig. 6, it can be observed that the sum rate performance ${ }^{4}$ increases with increasing $K$, even when MRC is performed with imperfect channel estimates (using the proposed uplink training sequence). The next relevant question is whether the behaviour of increasing sum rate with increasing $K$ (fixed $M$ ) is still true with phase noise. It turns out that this is no more true, as can be seen from Fig. 6. To be precise, the achievable sum rate initially increases with increasing $K$ and then decreases with further increase in $K$. The explanation for this observation is as follows. The initial increase in the achievable sum rate is due to the fact that more users are multiplexed on the same frequency-time resource. However when $K$ becomes large, the duration of the proposed training phase is long due to which there is partial loss of phase coherency between the channel estimates and the received symbols during data phase. For a sufficiently large $K$, the corresponding loss in phase coherency negatively impacts the multiplexing gain offered by having a large number of users.

\section{CONCLUSIONS}

We investigated the effect of oscillator phase noise in the sum rate performance of a frequency selective uplink MUMIMO channel with imperfect channel knowledge as the

\footnotetext{
${ }^{4}$ Observe that the no phase noise curve is generated by (15) (limiting value as $N_{D} \rightarrow \infty$ )
} 


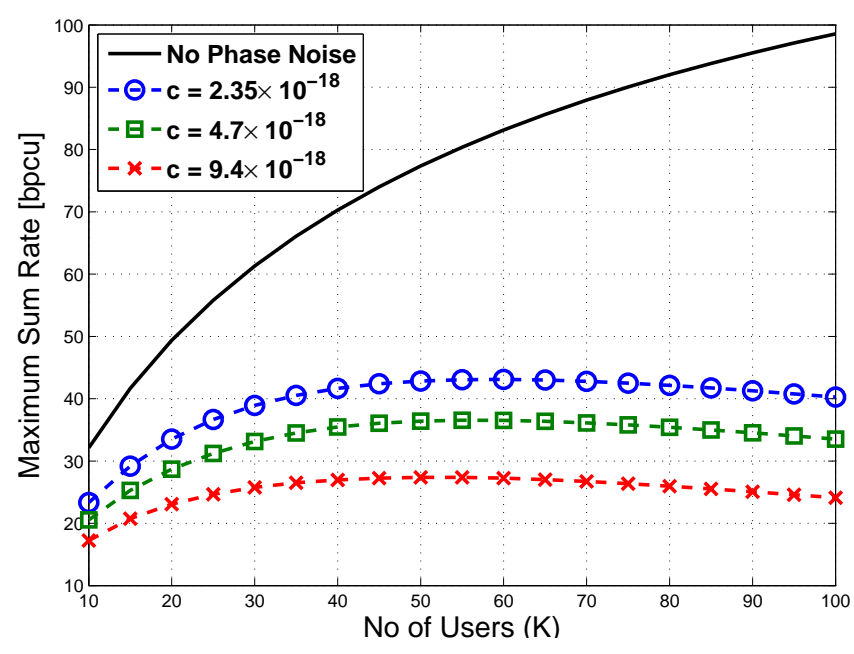

Fig. 6. Maximum achievable sum rate [bpcu] as a function of the number of users, $K$, for various values of the local oscillator parameter $c$, as defined in Section II-A.

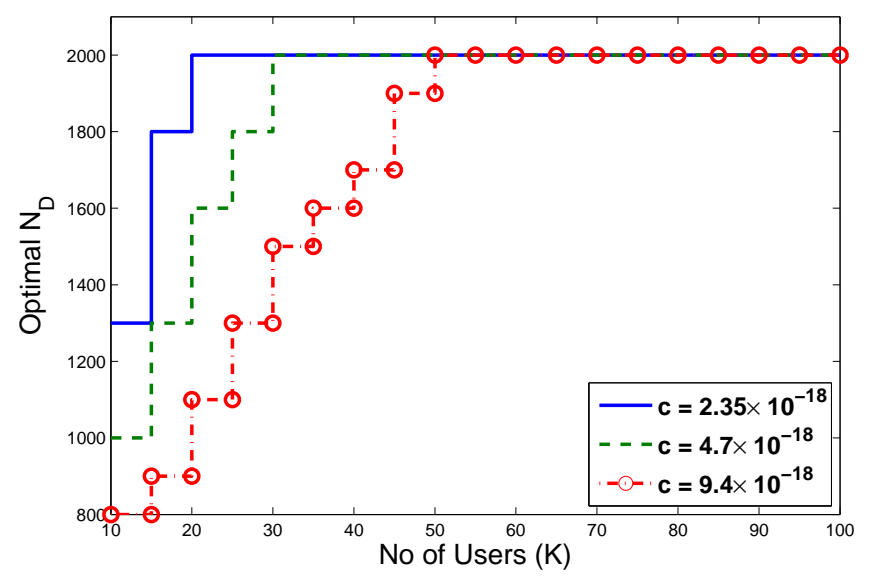

Fig. 7. Optimal value of $N_{D}$ (where the achievable sum rate is maximized) as a function of the number of users, $K$. The optimal value of $N_{D}$ for the no phase noise case is unbounded (i.e. $\infty$ ) for any $K$.

number of BS antennas grows large. We proposed a low complexity channel estimation and detection scheme and derived a closed form expression on the achievable sum rate. Based on that, we showed that an $O(\sqrt{M})$ array gain is achievable in the presence of phase noise. However there is an information rate loss due to phase noise, which is more significant at high spectral efficiencies and when the time interval utilized for data transmission grows large. Further, increasing the number of users does not result in an ever increasing sum rate performance, since the time interval required for training becomes large resulting in partial loss of coherency between the received symbols and the channel estimates. The results shown here depend on the simplistic training scheme we considered. The main motivation for the choice of this channel estimation scheme was to facilitate the derivation of the lower bound on the sum rate. However, we expect that a more sophisticated channel estimation scheme will have a marginal effect on the final conclusions.

\section{APPENDIX \\ ProOF OF THEOREM 1}

Proof: We start the proof by calculating the constant $\mathbb{E}\left[A_{k}[i]\right]$. We have

$$
\begin{aligned}
\mathbb{E} & {\left[A_{k}[i]\right]=\mathbb{E}\left[\sqrt{P_{D}} \sum_{m=1}^{M} \sum_{l=0}^{L-1}\left|g_{m, k, l}\right|^{2} e^{-j(\phi[i+l]-\phi[(k-1) L+l])}\right.} \\
\cdot & \left.e^{-j\left(\theta_{k}[(k-1) L]-\theta_{k}[i]\right)}\right]=\sqrt{P_{D}} \sum_{m=1}^{M} \sum_{l=0}^{L-1} \mathbb{E}\left[\left|g_{m, k, l}\right|^{2}\right] \\
\cdot & \mathbb{E}\left[e^{-j(\phi[i+l]-\phi[(k-1) L+l])}\right] \mathbb{E}\left[e^{-j\left(\theta_{k}[(k-1) L]-\theta_{k}[i]\right)}\right] \\
= & \sqrt{P_{D}} M e^{-4 \pi^{2} f_{c}^{2} c T_{s}(i-(k-1) L)}
\end{aligned}
$$

where we have used the fact the channel realizations, $g_{m, k, l}$, the phase noise at the $\mathrm{BS}, \phi$, and the phase noise at the $k$-th user, $\theta_{k}$, are mutually independent random processes. Additionally, as mentioned in the text, the phase noise processes at the users and the base station are assumed to be independent Wiener processes with independent Gaussian increments. Consequently, after a time interval, $\Delta t$, the phase drift of an oscillator is a zero mean Gaussian random variable with variance that is proportional to $\Delta t$. That is,

$$
\begin{aligned}
w_{\phi}[i-(k-1) L] & \triangleq \phi[i+l]-\phi[(k-1) L+l] \\
& \sim \mathcal{N}\left(0,4 \pi^{2} f_{c}^{2} c T_{s}(i-(k-1) L)\right) \\
w_{\theta_{k}[i-(k-1) L]} & \triangleq \theta_{k}[i]-\theta_{k}[(k-1) L] \\
& \sim \mathcal{N}\left(0,4 \pi^{2} f_{c}^{2} c T_{s}(i-(k-1) L)\right) .
\end{aligned}
$$

Therefore, we compute $\mathbb{E}\left[e^{-j w_{\phi}[i-(k-1) L]}\right]=\varphi_{\phi}(-1)=$ $e^{-2 \pi^{2} f_{c}^{2} c T_{s}(i-(k-1) L)}$ and $\mathbb{E}\left[e^{j w_{\theta_{k}}[i-(k-1) L]}\right]=\varphi_{\theta_{k}}(1)=$ $e^{-2 \pi^{2} f_{c}^{2} c T_{s}(i-(k-1) L)}$, where $\varphi_{\phi}$ and $\varphi_{\theta_{k}}$ are the characteristic functions of the zero mean Gaussian random variables $w_{\phi}[i-(k-1) L]$ and $w_{\theta_{k}}[i-(k-1) L]$, respectively. This concludes the calculation of $\mathbb{E}\left[A_{k}[i]\right]$.

We proceed with the calculation of the variance of the effective noise term, $\mathrm{EN}_{k}[i]$.

$$
\begin{aligned}
& \operatorname{Var}\left(\mathrm{EN}_{k}[i]\right) \triangleq \mathbb{E}\left[\left|\mathrm{EN}_{k}[i]-\mathbb{E}\left[\mathrm{EN}_{k}[i]\right]\right|^{2}\right] \\
& \quad=\operatorname{Var}\left(\left(A_{k}[i]-\mathbb{E}\left[A_{k}[i]\right]\right) x_{k}[i]\right)+\operatorname{Var}\left(\operatorname{ISI}_{k}[i]\right) \\
& \quad+\operatorname{Var}\left(\operatorname{MUI}_{k}[i]\right)+\operatorname{Var}\left(\operatorname{AN}_{k}[i]\right)
\end{aligned}
$$

In the last step we have used the fact that the terms in $\mathrm{EN}_{k}[i]$ are mutually uncorrelated. We start with the calculation of the variance of the additional interference $\left(A_{k}[i]-\mathbb{E}\left[A_{k}[i]\right]\right) x_{k}[i]$,

$$
\begin{aligned}
\mathbb{E} & {\left[\left|\left(A_{k}[i]-\mathbb{E}\left[A_{k}[i]\right]\right) x_{k}[i]\right|^{2}\right]=\mathbb{E}\left[\left|A_{k}[i]\right|^{2}\right]-\left(\mathbb{E}\left[A_{k}[i]\right]\right)^{2} } \\
= & P_{D} \sum_{m=1}^{M} \sum_{l=0}^{L-1} \mathbb{E}\left[\left|g_{m, k, l}\right|^{4}\right]+P_{D} \sum_{m=1}^{M} \sum_{\substack{l=0 \\
l=0}}^{L-1} \sum_{\substack{l^{\prime}=0 \\
l^{\prime} \neq l}}^{L-1} \mathbb{E}\left[\left|g_{m, k, l}\right|^{2}\right] \\
\cdot & \mathbb{E}\left[\left|g_{m, k, l^{\prime}}\right|^{2}\right] \mathbb{E}\left[e^{-j\left(\phi[i+l]-\phi\left[i+l^{\prime}\right]-\phi[(k-1) L+l]+\phi\left[(k-1) L+l^{\prime}\right]\right)}\right]
\end{aligned}
$$




$$
\begin{aligned}
& +P_{D} \sum_{m=1}^{M} \sum_{\substack{m^{\prime}=1 \\
m^{\prime} \neq m}}^{M} \sum_{l=0}^{L-1} \sum_{l^{\prime}=0}^{L-1} \mathbb{E}\left[\left|g_{m, k, l}\right|^{2}\right] \mathbb{E}\left[\left|g_{m^{\prime}, k, l^{\prime}}\right|^{2}\right] \\
& \cdot \mathbb{E}\left[e^{-j\left(\phi[i+l]-\phi\left[i+l^{\prime}\right]-\phi[(k-1) L+l]+\phi\left[(k-1) L+l^{\prime}\right]\right)}\right] \\
& -P_{D} M^{2} e^{-8 \pi^{2} f_{c}^{2} c T_{s}(i-(k-1) L)}=P_{D} M \sum_{l=0}^{L-1} 2 d_{k, l}^{2} \\
& +P_{D} M \sum_{l=0}^{L-1} \sum_{l^{\prime}=0}^{L-1} d_{k, l} d_{k, l^{\prime}} e^{-4 \pi^{2} f_{c}^{2} c T_{s}\left|l-l^{\prime}\right|} \\
& +P_{D} M(M-1) \sum_{l=0}^{L-1} \sum_{l^{\prime}=0}^{L-1} d_{k, l} d_{k, l^{\prime}} e^{-4 \pi^{2} f_{c}^{2} c T_{s}\left|l-l^{\prime}\right|} \\
& -P_{D} M^{2} e^{-8 \pi^{2} f_{c}^{2} c T_{s}(i-(k-1) L)} \\
& =P_{D} M^{2} P_{\mathrm{pn}}+P_{D} M \sum_{l=0}^{L-1} d_{k, l}^{2},
\end{aligned}
$$

where $P_{\mathrm{pn}} \triangleq \sum_{l=0}^{L-1} \sum_{l^{\prime}=0}^{L-1} d_{k, l} d_{k, l^{\prime}} e^{-4 \pi^{2} f_{c}^{2} c T_{s}\left|l-l^{\prime}\right|}-$ $e^{-8 \pi^{2} f_{c}^{2} c T_{s}(i-(k-1) L)}$. The variance of the ISI term can be computed by

$$
\begin{aligned}
& \mathbb{E}\left[\left|\operatorname{ISI}_{k}[i]\right|^{2}\right]=\mathbb{E}\left[\mid \sqrt{P_{D}} \sum_{m=1}^{M} \sum_{\substack { l=0 \\
\begin{subarray}{c}{q=0 \\
q \neq l{ l = 0 \\
\begin{subarray} { c } { q = 0 \\
q \neq l } }\end{subarray}}^{L-1} \sum_{m, k, l}^{L-1} g_{m, k, q}\right. \\
& \left.\left.e^{-j(\phi[i+l]-\phi[(k-1) L+l])+\theta_{k}[(k-1) L]-\theta_{k}[i+l-q]} x_{k}[i+l-q]\right|^{2}\right] \\
& =P_{D} \sum_{m=1}^{M} \sum_{l=0}^{L-1} \sum_{\substack{q=0 \\
q \neq l}}^{L-1} d_{k, l} d_{k, q}=P_{D} M \sum_{l=0}^{L-1} d_{k, l}\left(1-d_{k, l}\right) \\
& =P_{D} M\left(1-\sum_{l=0}^{L-1} d_{k, l}^{2}\right)
\end{aligned}
$$

where we have used the normalization (1), the fact that the transmitted symbols $x_{k}[i]$ are temporally independent and the assumptions on the statistical properties of the channel realizations and the phase noise processes. For the multi-user interference, based on the statistical properties of the channels, phase noise processes, transmitted information symbols and the PDP normalization (1), we can calculate

$$
\begin{aligned}
& \mathbb{E}\left[\left|\mathrm{MUI}_{k}[i]\right|^{2}\right]=\mathbb{E}\left[\mid \sqrt{P_{D}} \sum_{m=1}^{M} \sum_{\substack{p=1 \\
p \neq k}}^{K} \sum_{l=0}^{L-1} \sum_{q=0}^{L-1} g_{m, k, l}^{*} g_{m, p, q}\right. \\
& \left.\left.e^{-j(\phi[i+l]-\phi[(k-1) L+l])+\theta_{k}[(k-1) L]-\theta_{p}[i+l-q]} x_{p}[i+l-q]\right|^{2}\right] \\
& =P_{D} \sum_{m=1}^{M} \sum_{\substack{p=1 \\
p \neq k}}^{K} \sum_{l=0}^{L-1} \sum_{q=0}^{L-1} d_{k, l} d_{p, q}=P_{D} M(K-1)
\end{aligned}
$$

We conclude the proof with the calculation of the additive noise power

$$
\begin{aligned}
& \mathbb{E}\left[\left|\mathrm{AN}_{k}[i]\right|^{2}\right]=\frac{P_{D}}{P_{p} K L} \sum_{m=1}^{M} \sum_{m^{\prime}=1}^{M} \sum_{p=1}^{K} \sum_{p^{\prime}=1}^{K} \sum_{l=0}^{L-1} \sum_{l^{\prime}=0}^{L-1} \sum_{a=1-L}^{L-1} \\
& \mathbb{E}\left[g_{m, p, l-a} g_{m^{\prime}, p^{\prime}, l^{\prime}-a} e^{-j\left(\phi[i+l]-\phi\left[i+l^{\prime}\right]-\theta_{p}[i+a]+\theta_{p^{\prime}}[i+a]\right)}\right. \\
& \left.\cdot x_{p}[i+a] x_{p^{\prime}}^{*}[i+a] n_{k}[(k-1) L+l] n_{k}\left[(k-1) L+l^{\prime}\right]\right] \\
& +\sum_{m=1}^{M} \sum_{l=0}^{L-1} \mathbb{E}\left[\left|\hat{g}_{m, k, l}\right|^{2}\right]=\frac{P_{D} \sigma^{2}}{P_{p} K L} \sum_{m=1}^{M} \sum_{p=1}^{K} \sum_{l=0}^{L-1} \sum_{a: l-a=0}^{L-1} d_{p, l-a} \\
& +\sigma^{2} \sum_{m=1}^{M} \sum_{l=0}^{L-1}\left(\frac{\sigma^{2}}{P_{p} K L}+\mathbb{E}\left[\left|g_{m, k, l}\right|^{2}\right]\right)=\sigma^{2} \frac{P_{D} M}{P_{p}} \\
& +\sigma^{2} M \sum_{l=0}^{L-1}\left(\frac{\sigma^{2}}{P_{p} K L}+d_{k, l}\right)=\sigma^{2} M\left(\frac{P_{D}}{P_{p}}+\frac{\sigma^{2}}{P_{p} K}+1\right) .
\end{aligned}
$$

\section{REFERENCES}

[1] D. Gesbert, M. Kountouris, R. W. Heath Jr., C.-B. Chae, and T. Sälzer, "Shifting the MIMO Paradigm," IEEE Signal Processing Magazine, vol. 24, pp. $36-46$, September 2007.

[2] T. L. Marzetta, "Noncooperative cellular wireless with unlimited numbers of base station antennas," Wireless Communications, IEEE Transactions on, vol. 9, pp. 3590-3600, Nov. 2010.

[3] H. Q. Ngo, E. G. Larsson, and T. L. Marzetta, "Energy and spectral efficiency of very large multiuser MIMO systems," Communications, IEEE Transactions on, submitted, vol. arXiv:1112.3810, 2011.

[4] A. Pitarokoilis, S. K. Mohammed, and E. G. Larsson, "On the optimality of single-carrier transmission in large-scale antenna systems," Wireless Communications Letters, IEEE, accepted for publication.

[5] T. Pollet, M. Van Bladel, and M. Moeneclaey, "BER sensitivity of OFDM systems to carrier frequency offset and wiener phase noise," Communications, IEEE Transactions on, vol. 43, pp. $191-193$, feb/mar/apr 1995.

[6] L. Tomba, "On the effect of Wiener phase noise in OFDM systems," Communications, IEEE Transactions on, vol. 46, pp. 580 -583, May 1998.

[7] I. Barhumi, G. Leus, and M. Moonen, "Optimal training design for MIMO OFDM systems in mobile wireless channels," Signal Processing, IEEE Transactions on, vol. 51, pp. 1615 - 1624, June 2003.

[8] S. Wu and Y. Bar-Ness, "OFDM systems in the presence of phase noise: consequences and solutions," Communications, IEEE Transactions on, vol. 52, pp. 1988 - 1996, Nov. 2004.

[9] A. Demir, A. Mehrotra, and J. Roychowdhury, "Phase noise in oscillators: a unifying theory and numerical methods for characterization," Circuits and Systems I: Fundamental Theory and Applications, IEEE Transactions on, vol. 47, pp. 655-674, May 2000.

[10] D. Petrovic, W. Rave, and G. Fettweis, "Effects of phase noise on OFDM systems with and without PLL: Characterization and compensation," Communications, IEEE Transactions on, vol. 55, pp. 1607 -1616 , Aug. 2007.

[11] B. Hassibi and B. Hochwald, "How much training is needed in multiple-antenna wireless links?," Information Theory, IEEE Transactions on, vol. 49, pp. 951 - 963, Apr. 2003.

[12] T. L. Marzetta, "How much training is required for multiuser MIMO?," in Fortieth Asilomar Conference on Signals, Systems and Computers, 2006. ACSSC '06., pp. 359 -363, November 2006.

[13] D. Petrovic, W. Rave, and G. Fettweis, "Common phase error due to phase noise in OFDM-estimation and suppression," in Personal, Indoor and Mobile Radio Communications, 2004. PIMRC 2004. 15th IEEE International Symposium on, vol. 3, pp. 1901 - 1905 Vol.3, Sept. 2004.

[14] H. Q. Ngo, E. G. Larsson, and T. L. Marzetta, "Uplink power efficiency of multiuser MIMO with very large antenna arrays," in Proc. of Allerton Conference on Communication, Control, and Computing, Sep. 2011. 